\title{
On Neutrino Oscillations and Predicting the 125 GEV Two Photon Emission State from p-p Collisions Based on the 5D Homogeneous Space-Time Projection Model
}

\author{
K. W. Wong ${ }^{1}$, G. Dreschhoff ${ }^{1}$, H. Jungner ${ }^{2}$ \\ ${ }^{1}$ Department of Physics and Astronomy, University of Kansas, Lawrence, USA \\ ${ }^{2}$ Radiocarbon Dating Lab, University of Helsinki, Helsinki, Finland \\ Email: kww88ng@gmail.com, giselad@ku.edu, hogne.jungner@helsinki.fi
}

Received August 8, 2012; revised September 11, 2012; accepted September 18, 2012

\begin{abstract}
Previously the 5D homogeneous space-time metric was introduced with explicitly given projection operators in matrix form which map the 5D space-time manifold into a Lorentzian space-time. Based on this projection model, vector field and spinor solutions are found to be expressible in terms of $\mathrm{SU}(2) \mathrm{xL}$ and $\mathrm{SU}(3) \mathrm{xL}$, where $\mathrm{L}$ is the $4 \mathrm{D}$ Lorentz space-time group. The spinor solutions give the SU(2) leptonic states arising from space-time projection, whereas the $\mathrm{SU}(3)$ representation arises from conformal projection and gives the quarks, and due to gauge requirement leads to mesons and baryons. This process of mapping the 5D space-time manifold into the 4D space-time is at the basis of an analysis of the recent CERN experimental results, the presence of neutrino oscillations and the observed $125 \mathrm{GeV}$ resonance in the p-p collisions, respectively. In fact, it is found that the spinor solution contains an oscillating phase, and the $125 \mathrm{GeV}$ resonance is shown to be predictable, thereby 1) eliminating the need to introduce a Higgs vacuum, and 2) can be shown possibly to be an indicator for a missing heavy baryon octet.
\end{abstract}

Keywords: Neutrino Oscillation; $125 \mathrm{GeV}$ p-p Bound State; Hadron Mass Levels; 5D Homogeneous Space-Time

\section{Introduction}

Recently there was some excitement within the physics community related to results of two fundamental experimental findings. By far the more publicized is the finding of a $125 \mathrm{GeV}$ two photon emission obtained from the p-p collision experiment done by the CERN Large Hadron Collider [1,2] and the Tevatron [3]. It is billed as an indication of the existence of the Higgs Boson, which was advanced some half a century ago by Higgs [4] for giving the quarks of Gell-Mann's standard model [5] their basic masses. It is clear that in the 4D space-time domain, mass cannot be generated by interactions of mass-less fields. The second experimental finding is the oscillation of the neutrino states [6-8], making the three leptonic neutrinos able to evolve into each other on their propagation through space-time. It is these two interesting results that we like to discuss in this paper. Not long ago, we proposed a 5D homogeneous space-time domain [9], and showed that we can derive the quarks of the standard model by imposing a projection action. In fact, this projection can be separated into a pure space to time axis projection $\mathrm{P}_{0}$, which would necessarily produce the leptons plus their corresponding neutrinos as given by the
$\mathrm{SU}(2)$ representation. The space-space projection $\mathrm{P}_{1}$ is a conformal projection, and readily gives the $\mathrm{SU}(3)$ quarks, with fractional charges and with corresponding intrinsic masses. Therefore, this 5D theory requires no Higgs Bosons to give quarks their masses. Hence if the observed $125 \mathrm{GeV}$ two photon emission state cannot be explained by our $5 \mathrm{D}$ model, then perhaps this result would be more convincing that it is a verification of the Higgs field existence. We separate our discussions into three parts. For the first part, we show explicitly, the neutrino oscillation as obtained by the 5D projection theory [9]. In the second part, we will show that according to our theory and the $\mathrm{SU}(3)$ representations, there should exist a yet not discovered very high energy octet representation of the baryons. It is also pointed out that due to the extremely large masses of these eight baryons, they could be nearly degenerate, if the mass splitting between them is negligibly small. In fact then the total sum for a pair of all eight baryon masses also amounts to the order of $120 \mathrm{GeV}$ accidentally, but might not be correlated to the p-p result. From there in the third part, we put forward a discussion on the $125 \mathrm{GeV}$ resonance created by the p-p collision, which is suggested to be predictable from the $5 \mathrm{D}$ projection model, and perhaps has no correlation to the exis- 
tence of a Higgs field.

\section{The Neutrino Solution}

The supposition that the universe is in the 5D homogeneous space-time manifold might be verifiable from mass-less fields that are solutions of the 5D metric operator via boundary conditions in the 4D coordinate volume projected into the Lorentz 4D space-time domain that they must satisfy. Of these fields, there are the photons, e-trinos, and neutrinos. The photons satisfy the 4D Maxwell operator, hence cannot provide boundary conditions that are in 5D, while the e-trinos can only exist in 5D and not in Lorentz 4D, thus cannot be observed by us that live in the Lorentz 4D domain. We are therefore confined to studying the solutions of the neutrinos.

The neutrino is a solution of a $P_{0}$ projection on the $5 \mathrm{D}$ spinor, as given by

$$
\tilde{\Psi}=\mathrm{e}^{-\mathrm{i} \alpha p_{4} \Phi} \Psi
$$

where $\Phi=2 \pi / q$ is the quantum flux for a charge " $q$ ". Note " $q$ " is not necessary " $e$ ", it is simply a coupling constant between the spinor and the vector potential fields, as we have discussed in ref. [9], $\alpha$ is a finite constant $=q / m$, and $\Psi$ is a vector field independent spinor in 5D space-time manifold, via gauge transformation. It should be noted that $\Phi=\oint A_{\mu} \mathrm{d} x^{\mu}=2 \pi / q$, where $A_{\mu}$ is in the 4D Maxwell space-time, must go to infinity as $q$ goes to zero, meaning the charge-less spinor is not coupled to the vector fields in the 4D Hilbert subspace of the (4x1)D manifold, thus the flux loop must enclose the entire 4D Hilbert subspace of the universe.

It is then obvious that $P_{0}$ produces the $\mathrm{SU}(2)$ group, with $q=e$ or 0 . For $q=e$, we have the Dirac equation for a charged massive spinor. However if $q=0$, we must have $m=0$, such that $q / m$ can remain a constant. In order that the phase factor in Equation (2.1) is properly defined we also must have the limit $\Phi p_{4}=2 \pi / \alpha$. Since $\Phi$ diverges as " $q$ " goes to zero, $p_{4}$ must simultaneously go to zero. It is this solution that makes the neutrino both charge-less and mass-less. It is easy to see without $P_{0}$ action, it is a 5D plane wave, without boundary [9].

The neutrino equation in Lorentz space-time is not simply eliminating $m$ from the Dirac equation as QED will have. Actually, the neutrino, being charge-less, is in the 4D Lorentz energy-momentum space without making a $P_{0}$ projection. The $4 \mathrm{D}$ Lorentz energy-momentum space is different from the 4D Lorentz space-time, as fixing the rest mass equal to zero, implies from Fourier transformation fixing $x_{4}$ equal to infinity. However the 5D homogeneous space-time metric makes all coordinates finite at a fixed time $t$, thus this requirement cannot be satisfied. On the other hand, the 5D homogeneous metric operator implies all field solutions of it must be mass-less. These contradictions must be due to not imposing the boundary conditions to the field solutions. Or that these 5D fields must satisfy boundary conditions on the boundary of the universe as defined by the time $t$ of its absolute age. Therefore to find it, we return to the spinor equation given in the 5D homogeneous space-time manifold.

$$
\sum_{\mu=0}^{4} \tilde{\gamma}_{\mu} \tilde{\partial}_{\mu} \tilde{\Psi}=0
$$

where we now transform the 5D spinor into the form given by Equation (2.1).

The linear 5D operator can be explicitly expressed in terms of two additive terms. The first term is purely that due to the $x_{4}$ component, while the second term is just the 4D Dirac operator without mass.

$$
\tilde{\gamma}_{4} \partial_{4} \tilde{\Psi}+\sum_{\mu=0}^{3} \tilde{\gamma}_{\mu} \partial_{\mu} \tilde{\Psi}=0
$$

as the phase factor is subject to the first term operator. In order to perform the differentiation, we make the Fourier transformation to $p_{4}$, we get for the first term

$$
\tilde{\gamma}_{4} \partial_{4} \mathrm{e}^{-\mathrm{i} \alpha p_{4} \Phi} \Psi=\left[-\mathrm{i} \alpha \frac{\Phi}{x_{4}^{2}} \Psi+\left(\partial_{4} \Psi\right)\right] \mathrm{e}^{-\mathrm{i} \alpha p_{4} \Phi} \tilde{\gamma}_{4}
$$

Substituting Equation (2.4) into Equation (2.3) we obtain

$$
\left[\sum_{\mu=0}^{4} \tilde{\gamma}_{\mu} \tilde{\partial}_{\mu}-\mathrm{i} \omega_{4} \tilde{\gamma}_{4}\right] \Psi=0
$$

where $\omega_{4}=\frac{\alpha \Phi}{x_{4}^{2}}=\frac{\alpha}{x_{4}^{2}} \oint A_{\mu} \mathrm{d} x^{\mu}$.

There are 3 independent loop planes in a volume of 4D coordinates, as depicted by a doughnut 3D figure instead of a 3D homogeneous sphere following Perelman's proof of the Poincaré conjecture. Hence the quantum flux actually is a pseudo vector in the 4D coordinate space. From the 5D metric $x_{4}^{2}=T^{2}-\boldsymbol{x}^{2}$, where $T$ is the age of the universe. As $T$ increases to a very large value, the loop length for the flux will also. Hence it is possible that $\omega_{4}$ is finite. In fact for the boundary condition to be unchanged with $T, \omega_{4}$ must be constant. To show that fixed $\omega_{4}$ gives the needed boundary condition on the mass-less fields in the finite universe, let us reformulate the $\omega_{4}$ constraint

$$
\frac{\omega_{4}}{\alpha} x_{4}^{2}=\oint A_{\mu} \mathrm{d} x^{\mu}
$$

where $\mu=0,1,2,3$.

From the 5D metric $x_{4}^{2}=t^{2}-x^{2}=\sum_{\mu=0}^{3} x_{\mu} x^{\mu}$.

By differentiating Equation (2.6) with $\partial_{\mu}$, Equation 
(2.6) becomes

$$
A_{\mu}=\frac{1}{\pi} \frac{\omega_{4}}{\alpha} x_{\mu}
$$

This simple solution of $A_{\mu}$ implies several philosophical implications:

I. The vector potential $A_{\mu}$ increases linearly with the expansion of the Lorentzian manifold as given by $x_{\mu}$ irrespective of any projection of the $x_{4}$ dimension.

II. Such an increase in $A_{\mu}$ as the 5D homogeneous space-time expands means the mass-less vector and spinor field energies also increase in proportion.

III. At the Lorentzian space-time boundary the vector potential $A_{\mu}(R)$ is given by

$$
A_{\mu}(R)=\frac{1}{\pi} \frac{\omega_{4}}{\alpha} R_{\mu}
$$

As there cannot be $A_{\mu}$ outside the 5D manifold or $A_{\mu} A^{\mu}$ must be zero on the surface enclosing the 5D domain as given by the 4D Maxwellian space-time. Corollary, it means $R_{\mu} R^{\mu}=0$, which is at the boundary of the 5D universe as depicted by $R_{\mu}$, and there can be no vector potential field, or no sources for the vector potentials as given by charged spinors, and no light reflection. In other words, the boundary of the universe is not observable. Since no fields can exist beyond the 5D manifold Equation (2.8) represents the fixed boundary condition for $A_{\mu}$ as well as that for the neutrino, which is not coupled to $A_{\mu}$ within $R_{\mu}$. As $R_{\mu} R^{\mu}=0$ maps the boundary, it is obvious that if $t$ is unidirectional, then the coordinate boundary is given by a spherical surface with radius $c t$.

IV. If the Lorentzian universe, which is created by the projection of the 5D homogeneous space-time manifold has a 3D space volume in the form of a doughnut as derived by Perelman, then such a $3 \mathrm{D}$ volume has 3 independent normals, namely 1) a normal to the surface of the doughnut, 2) a normal to the cross-section of the doughnut tube, 3) a normal to the doughnut ring crosssection. When $\boldsymbol{R}$ is transformed into a sphere, the sphere has more than just a single normal as depicted by the radius vector, but also an up and down orientation and Equation (2.8) is split into 3 coordinate vector equations with their respective different $\omega_{4}$ values. Hence it is very important to keep $q$ finite first and solve the equation before taking the limit $q$ going to zero! By keeping $\alpha$ finite, the solution to Equation (2.5) is a spin-space oscillator which satisfies the space-time metric, that is valid at any $T$, in the finite $4 \mathrm{D}$ space. This is the origin of this proper limiting procedure that will give us the 3 sets of charged massive leptons with their associated neutrinos due to the 3 remaining momentum space degrees of freedom. We can now rewrite the neutrino equation under $P_{0}$ projection, we get with $p_{4}=0$

$$
\left[\sum_{\mu=0}^{3} \gamma_{\mu} \partial_{\mu}-\left(P_{0} \mathrm{i} \omega_{4} \tilde{\gamma}_{4}\right)\right] \Psi_{v}=0
$$

where $P_{0} \omega_{4} \tilde{\gamma}_{4}$ is the PC violating neutrino oscillation energy. This limiting procedure of making neutrinos mass-less brings its solution back to a simple plane wave. Each neutrino is associated with a massive lepton. In the matter universe, the leptons can exist up to the boundary of the finite 4D Lorentz space-time domain, thus the neutrino solution need to be put into the exponentially oscillating form which must satisfy the boundary before taking the limit.

$$
\Psi_{v}=\mathrm{e}^{-\mathrm{i}\left[v+\left(P_{0} \omega_{4} \tilde{\gamma}_{4}\right)\right] t} \Psi_{v}(\boldsymbol{x})
$$

The 5D gamma matrices can be easily constructed from the Dirac 4D gamma matrices, which can be constructed in terms of spin matrices [9], and will not be presented here.

It should be noted that the neutrino exist in the $5 \mathrm{D}$ homogeneous space-time manifold, and only when gauge transformation is enacted the spin-space oscillation is created, and thus split into 3 neutrinos coupled to the 3 massive leptons.

As a specific lepton and its corresponding neutrino is created within a $3 \mathrm{D}$ spherical volume at time $t$, through Perelman's reverse mapping this neutrino state in question would evolve into the superposition of the 3 distinct cross-section loops due to the doughnut structure of the universe at its boundary as given by the age of the universe as depicted by the 5D metric [9]. It is this continuous growing of the $5 \mathrm{D}$ universe that introduces the oscillating factor into the evolving neutrino state with time from one into another among the three states as given by $\mathrm{SU}(2)$ representation.

An anti-lepton within the matter universe could not survive through all space before being annihilated. Hence, the anti-neutrino associated with this anti-lepton must also be confined to a very limited space domain that confines the anti-lepton. In another word the anti-neutrino associated with the anti-lepton, its coordinate oscillating frequency $\omega_{4}$ will become zero as the flux $\Phi$ must be finite due to a finite loop, like in a lab environment, while $T^{2}-\boldsymbol{x}^{2}$ approaches infinity with $\boldsymbol{x}^{2}$ being finitely confined and as $T$ increases to extremely large values. It is this physical picture that produces an a-symmetry between the neutrino and the anti-neutrino. Or conversely, it is the short ranged feature of the anti-neutrinos, caused by the annihilation of the anti-lepton that indicates we have a matter universe.

Since the neutrinos must have zero mass, by choosing the a-symmetry neutrino representation, they actually can cross over from one to the other as the anti-lepton annihilates. Choosing the limiting process that can only occur in the anti-particle domain will still maintain $\mathrm{SU}(2)$. As 
pointed out in recent experiments, the a-symmetry between leptons and anti-leptons was observed and it was suggested that such a-symmetry could be the reason we have a predominantly matter universe [6,7], therefore such finding is consistent with our 5D homogeneous space-time projection model.

\section{Fine Structure Mass Splitting in SU(3) Representations of Hadrons and Its Effect on Fixing the Gluon Potential Generated Mass Levels}

In our recent papers [9] and [10], we illustrated how the gluon potentials are derived through their strength factors from the Lorentz sum rules coupled with gauge invariance constraint and the restriction, that the quark constituents in the hadron must be Bohr-Sommerfeld quantized on the flux loop provided by the gauge constraint. However, as shown in these two papers, the numerical estimated values did not follow and obey these requirements exactly. As an illustration, we used a static model for the pion mass splitting in ref. [9], and deduced that the unit rest mass for the quark is $52.5 \mathrm{MeV}$, and hence obtained the pion gluon generated mass level as 129 $\mathrm{MeV}$. These values have to be somewhat inaccurate, as we can see that, based on the scaling of the gluon potential, the $\mathrm{K}$ mesons in the octet would need to have a mass greater than $516 \mathrm{MeV}$. The $\mathrm{K}$ meson masses are only around $494 \mathrm{MeV}$. Thus it is obvious that the unit strength scale of $129 \mathrm{MeV}$ is too large. Secondly, the BohrSommerfeld quantization requirement on the quark constituents in the pions implies that a static model is invalid. We will now show how these problems are removed.

According to the explicit meson-gluon potential form Equation (4.3) in ref. [9], the gluon generated mass level is inversely proportional to the loop radius $r^{2}$. In paper [10], we showed via Bohr-Sommerfeld quantization that

$$
r=\left(\hbar / m^{*} c\right) /\left\{C\left[C^{2}-1\right]^{0.5}\right\}
$$

where $C=1 /\left[1-(v / c)^{2}\right]^{0.5}$, and $m^{*}=\left(\left|s_{1}\right|+\left|s_{2}\right|\right) m=s m$, where $m$ is the unit quark rest mass.

Let the constituent relativistically independent gluon generated mass level per unit $m$ factor be proportional to $M_{0}$, then the corrected mass level becomes

$$
M=M_{0} C^{2}\left[C^{2}-1\right] s^{2}
$$

The bare quark relativistic mass $m^{\prime}$ is given by

$$
m^{\prime}=s m C
$$

Hence the meson mass $M^{*}$ is given by

$$
M^{* 2}=M^{2}+m^{\prime 2}
$$

Let us now consider the mass splitting between the charged pion and the neutral pion.

For the charged pion $s=1$, while for the neutral pion $s$ $<1$. If we denote the relativistic factor for the charged pions as $C$, and that of the neutral pions as $C^{\prime}$, it is obvious from Lenz's Law that we must have $C^{\prime}>C>1$.

With these formulas, we obtain the mass difference

$$
\begin{aligned}
(\mathrm{d} M(\pi))^{2}= & {\left[M^{*}(\text { charge })^{2}-M^{*}(\text { neutral })^{2}\right] } \\
= & M_{0}^{2}\left\{C^{4}\left(C^{2}-1\right)^{2}-C^{\prime 4}\left(C^{\prime 2}-1\right)^{2} \mathrm{~s}^{4}\right\} \\
& +m^{2}\left(C^{2}-\mathrm{s}^{2} C^{\prime 2}\right)
\end{aligned}
$$

Since the left hand side is positive, hence if $M_{0} \gg m$, as long as $C^{2}>C^{\prime 2} s^{2}$, and the coefficient for the $M_{0}$ term is very small, then there exist a consistent solution.

It is this requirement that guarantees the simultaneous gauge and Bohr-Sommerfeld constraint thus giving

$$
s^{2} C^{\prime 2}\left(C^{\prime 2}-1\right)=C^{2}\left(C^{2}-1\right)
$$

Under this equality the pion mass difference given by Equation (3.5), reduces to

$$
(\mathrm{d} M(\pi))^{2}=m^{2} C^{2}\left[1-\mathrm{d}^{2}\right]
$$

where $\mathrm{d}^{2}=\left(C^{2}-1\right) /\left(C^{\prime 2}-1\right)<1$.

The unit gluon potential induced mass is, therefore

$$
M=M_{0} C^{2}\left(C^{2}-1\right)
$$

For the $\mathrm{K}$ mesons, its gluon induced mass level is $4 M$, and the mass splitting is

$$
(\mathrm{d} M(\mathrm{~K}))^{2}=m^{2} C^{2}\left[1-\mathrm{d}^{\prime 2}\right]
$$

Hence, from the pions, combined with the $\mathrm{K}$ masses we obtain the mass level of $120 \mathrm{MeV}$ instead of the 129 $\mathrm{MeV}$ as given by the static model which does not obey Bohr-Sommerfeld quantization, as well as $m C$ and $\mathrm{d}$, $\mathrm{d}$ '. Note $m$ is not fixed by the quantization, as long as it is a constant, in agreement with the 5D homogeneous spacetime $P_{1}$ projection mathematical requirement [9].

It should be pointed out that this lower gluon mass level unit of $120 \mathrm{MeV}$ will eliminate the $\mathrm{K}$ meson mass problem we pointed out for the static model above. The pion mass difference is then purely dictated by $\mathrm{d}$ and making the Gell-Mann-Okubo mass formula valid, exactly in the same manner as that between the neutron and proton masses as we have discussed in paper [10]. With the new scale factor for the mass levels, we now adjust the meson SU(3) mass levels diagrams as shown in Figures 1(a) and (b).

It should be noted that there are no more inconsistencies with all the remaining mesons within the two known 


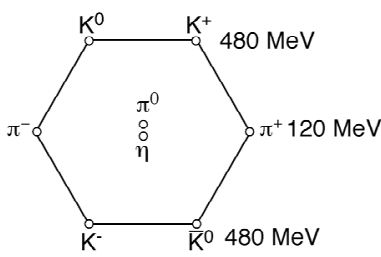

(a)

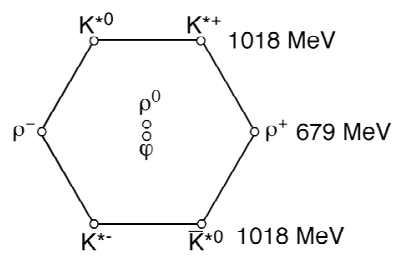

(b)
Figure 1. Consistent meson SU(3) mass level diagrams.

octets. As for the very heavy mesons, such as the $\mathrm{J} / \Psi$ and the Ys, which we have discussed in paper [10], their respective gluon generated mass levels must also be adjusted.

The $\mathrm{J} / \Psi$ gluon potential strength factor is obtained from the residual terms in the jet sum rule terms. We showed in paper [9], that it is $2(2 / 9)^{2}+(4 / 9)^{2}$. Hence its mass level is $2880 \mathrm{MeV}$ instead of the mass of $\mathrm{J} / \Psi$, which is $3096 \mathrm{MeV}$. Therefore, it means the constituent quark contribution to the mass is $216 \mathrm{MeV}$, a clear indication that the relativistic factor $\mathrm{C}$ is very large. The two $\mathrm{Y}$ mesons of $9460 \mathrm{MeV}$ and $4140 \mathrm{MeV}$ were assumed to be generated by the $(q q q q)$ intermediate quark currents. In fact, other earlier discovered heavy mesons, such as the $\mathrm{D}$, and $\mathrm{B}$ particles have been speculated as given by $\mathrm{SU}(6)$ representations [5]. In our model, we believe that the $(q q q q)$ currents can be approximated as the direct product between two (qq) currents. Since $\mathrm{Y}(4140)$ was observed to decay into $J / \Psi(3096)$ and $\varphi(1056)$, we can formulate the gluon potential strength factor as $\left\{2(2 / 9)^{2}+\right.$ $\left.(4 / 9)^{2}\right\} \times(9 / 9)^{2}+\left\{3(2 / 9)^{2}\right\} \times(9 / 9)^{2}$ such that the decay would conserve mass. Under this representation, we obtain the unit strength from the $4140 \mathrm{MeV}$ upper bound of $1.419 \mathrm{MeV}$. Hence in order to be able to include the bare quark constituents' contribution to the $\mathrm{Y}$ mass, we reduce the unit strength mass correspondence to $1.41 \mathrm{MeV}$ and obtain the quark mass term as $38 \mathrm{MeV}$. This modest contribution from the quark constituents makes this meson reasonably stable. The very heavy $\mathrm{Y}(9460)$ can only be generated by all the $(q q q q)$ current terms, or $(9 / 9)^{2}(9 / 9)^{2}$. Hence its gluon mass level is $9251 \mathrm{MeV}$ and the quark constituents are at $109 \mathrm{MeV}$. A detailed analysis of the $D$ and $B$ masses could further improve the estimation on the per unit strength mass correspondence. But it is clear that these quark potentials do not imply that the heavy mesons satisfy a $\mathrm{SU}(6)$ representation.

Following the simultaneous gauge and Bohr-Sommerfeld constraint analysis, by changing the $r$ dependence of the gluon potential, the baryon-gluon potentials unit strength scale that was given in ref. [9] and ref. [10] have to be reduced, as the baryon-gluon potential falls off as $r^{3}$. Therefore the proton, neutron mass level is given by

$$
M^{\prime}=M_{0}^{\prime} C^{3}\left(C^{2}-1\right)^{1.5} s(p)^{3}=M_{0}^{\prime} C^{\prime 3}\left(C^{\prime 2}-1\right)^{1.5} s(n)^{3}
$$

where $s(p)=5 / 3, s(n)=4 / 3$ and $C, C^{\prime}$ are the proton quarks and neutron quarks relativistic factors, respectively. $M_{0}^{\prime}$ is the $C, \mathrm{~s}$ independent factor of the gluon generated mass. It should be noted that the baryon masses do not fix the bare quark unit mass $m$, similar to the mesons. Hence as long as $m$ is a constant the theory is consistent. This conclusion has physical implication in that $m$ is like $e$ and $h$, a universal constant of the 5D homogeneous space-time projection theory.

It was found experimentally that the quark constituent in the proton contributed $11 \mathrm{MeV}$ to its mass [11,12], instead of the static model's $4 \mathrm{MeV}$, which is a direct proof to the Bohr-Sommerfeld quantization requirement. Based on this, we already know that the gluon potential induced mass has to be less than $934 \mathrm{MeV}$ provided by the static model. In order to get the $11 \mathrm{MeV}$ contribution from the quark mass, we find that the gluon induced mass level is $927.26 \mathrm{MeV}$. With this value we then deduced that the unit scale $(1 / 27)$ for the gluon strength is equivalent close to the value of $44.19 \mathrm{MeV}$ replacing the value of $44.5 \mathrm{MeV}$ obtained from the static model for the eigenvalue of the gluon repulsive potential [9]. This change might seem small, but is sufficient to eliminate some mass inconsistencies of certain baryons. The adjusted mass levels shown in Figure 2 of ref. [10] are re-illustrated in Figure 2.

It is interesting to observe that the mass level for the $\Sigma$ (Sigmas) in the octet representation is reduced from $1115 \mathrm{MeV}$ to $1105 \mathrm{MeV}$. This is clearly important for the mass consistency of the Lambda particle at the center, as its actual mass is only $1115.6 \mathrm{MeV}$ and therefore can not be consistent with a mass level of $1115 \mathrm{MeV}$, because the quark constituents contribution will always be greater than $0.6 \mathrm{MeV}$. A similar inconsistency mass problem arises for the $\Sigma$ (Sigmas) in the decuplet, without the reduction of the unit gluon mass scale.

The possible degenerate missing octet of heavy baryons can be analyzed as follows:

In order that this missing octet is nearly degenerate,

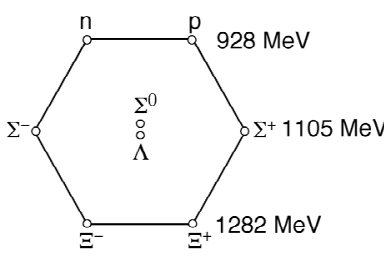

(a)

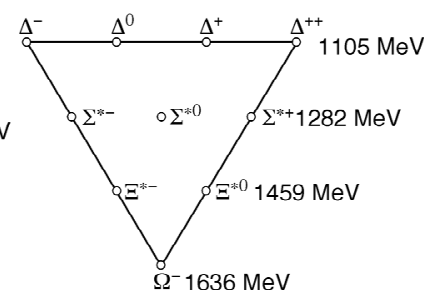

(b)
Figure 2. Self consistent baryon mass levels in the octet (a) and the decuplet (b). 
the three mass levels must be the same. From the sum rule, we obtained the remaining strength factors:

$$
3 A+5 B+3 C \text {. }
$$

This factor must be split into 3 identical levels plus 1 singlet value, and maintaining the sum rule:

Hence, we can rewrite these levels as

$$
\begin{gathered}
\text { Singlet. } 2 B-3 D \\
\text { Octet. } A+B+C+D
\end{gathered}
$$

such that

$$
\begin{aligned}
& A=(4 / 27)^{2} ; \\
& B=\left[(8 / 27)^{2}-2(2 / 27)^{2}\right] \\
& C=\left[(8 / 27)^{2}-2(1 / 27)^{2}\right] ; \\
& D=\left[a(4 / 27)^{2}+b(2 / 27)^{2}+c(1 / 27)^{2}\right],
\end{aligned}
$$

where $a, b, c$ are integers, such that $2 B>3 D$.

It could be seen that there is a wide range of choices.

For minimum, it would mean $D=0$.

For this case the total mass strength of the missing octet is

$$
8[A+B+C+D]
$$

In terms of $(1 / 27)^{2}$ producing a mass equivalent of 44.19 MeV, a pair of all the octet baryons will have a mass energy [A jet that balances momentum, but includes all the octet particles in pairs]:

$$
E=2 \times 44.19 \times 8[A+B+C+D](27)^{2},
$$

thus $E>2 \times 44.19(27)^{2} \times 8[A+B+C]$ or $2 \times 44.19 \times$ $8 \times 134=94.74 \mathrm{GeV}$ In order to get from $E=94.74$ to $125 \mathrm{GeV}, D$ has to contribute a factor of 42 to 43 . To get that, we can have $a=2, b=2, c=2$, or $c=3$. Which means $D$ has a strength factor equivalent of $2(16+4+1)$, or $2(16+4)+3 \times 1$. This estimation did not account for the inter-level mass splittings. However, due to the smaller values from the bare quark constituents contribution, it can only account for a small correction, no more than the $(1 / 27)^{2}$ factor would. Hence we will choose $D=\left[a(4 / 27)^{2}+b(2 / 27)^{2}+c(1 / 27)^{2}\right]$. This jet production, although having an energy similar in value as the 2 photons emission detected in the CERN experiment, is not the same process. In fact, the choice of $a=b=c=2$ produces $2 B<3 D$, which violates the rule that no baryon can have negative mass! To Adjust that, we can choose $a=2, b=1, c=0$ for example. It is instructive to rewrite the singlet and octet masses, due to this choice of the gluon strength factors. We get for the singlet:

$$
\begin{aligned}
& 2 B-3 D=2\left[(8 / 27)^{2}-2(2 / 27)^{2}\right] \\
& -3 \times 2\left[(4 / 27)^{2}+(2 / 27)^{2}+(1 / 27)^{2}\right] \\
& +3(2 / 27)^{2}+6(1 / 27)^{2} \\
& =2\left[(8 / 27)^{2}-3(4 / 27)^{2}-5(2 / 27)^{2}\right]+3(2 / 27)^{2} .
\end{aligned}
$$

This factor contains all the 4 distinct strength factors, while the degenerate octet levels are given by:

$$
\begin{aligned}
& A+B+C+D=\left[(4 / 27)^{2}\right]+\left[(8 / 27)^{2}-2(2 / 27)^{2}\right] \\
& +\left[(8 / 27)^{2}-2(1 / 27)^{2}\right]+\left[2(4 / 27)^{2}+2(2 / 27)^{2}\right] \\
& =2(8 / 27)^{2}+3(4 / 27)^{2}-(2 / 27)^{2}-2(1 / 27)^{2},
\end{aligned}
$$

which involves all 4 strength factor terms, $(8 / 27)^{2}$, $(4 / 27)^{2},(2 / 27)^{2}$ and $(1 / 27)^{2}$.

It is easy to estimate the singlet mass. We have $44.19 \times[64 \times 2-6 \times 16-10 \times 4+12]=44.19 \times 4$ or 176.8 $\mathrm{MeV}$. While the octet levels will give a total 2 octet baryon mass of $44.19 \times 2 \times 8 \times 170 \mathrm{MeV}$ or $118 \mathrm{GeV}$. It should be pointed out, that by changing $D$ slightly, we can get to $2 B$ being nearly equal to $3 D$, and such a singlet could be mistaken as a heavy lepton? While the degenerate octet jet will have a resonance at $120 \mathrm{GeV}$, but can never reach the $125 \mathrm{GeV}$ value.

The fact that the well known octet and decuplet only contain the three strength factors $(4 / 27)^{2},(2 / 27)^{2}$ and $(1 / 27)^{2}$, allows one to develop a theory based on chromodynamics that is valid for mesons, and perhaps might be the reason why we had difficulty identifying this degenerate remaining octet and the singlet experimentally, as the factor $(8 / 27)^{2}$ is unique to these baryon states only. Of course, it is totally possible that this missing octet is not degenerate, then the experimental resonance around $120 \mathrm{GeV}$ has no relationship to the missing octet.

In conclusion, we have shown that the relativistic mass corrections to the quark constituents due to the requirement of the simultaneous gauge and Bohr-Sommerfeld quantization are of vital importance to the proper determination of the gluon potentials generated mass levels in the $\mathrm{SU}(3)$ representations of hadrons, and it is not just for the lesser mass splitting between particles within the same mass level.

\section{Comments on the Observed $125 \mathrm{GeV}$ Two Photon Emission State from the p-p Scattering Experiment}

Recently physicists celebrated the finding of a 2 photons emission state at $125 \mathrm{GeV}$ obtained from the direct p-p scattering in the CERN Large Hadron Collider, as a possible verification of the existence of a Higgs Boson $[1,2]$. In this note, we would like to point out that there are al- 
ternative possibilities to explain this result without invoking the presence of a Higgs field.

It is well recognized that the proton, a composite of $2 u$ and 1d quarks, derives its mass from two factors: 1 ) The bare masses of the $2 u$ and $1 d$ quarks, held together by the gauge confinement. 2) This final composite mass is further significantly modified by the gluon fields acting between the quarks. The gluon fields are obviously of short range and might or might not be repulsive. Should they be repulsive, then they contribute to increasing the proton mass above the sum of the bare quark masses. In the p-p collision experiment, if an intermediate hadron state is formed, then it must consist of $4 \mathrm{u}$ and $2 \mathrm{~d}$ quarks. This composite carries a net charge of 2e. Hence the gauge transformation will give a quantum flux of $h / 2 \mathrm{e}$, instead of $\mathrm{h} / \mathrm{e}$. This state actually could be viewed as a complex Cooper pair state. Because of the reduction of the quantum flux by $1 / 2$, it is possible that it also reduces the $2 p$-hadron size $r$ by $1 / 2$. Hence if the gluon fields are repulsive, and vary as $(1 / \mathrm{r})^{\mathrm{n}}$, then it would follow that the resultant mass contribution from them would increase by the order $2 \times(2)^{\mathrm{n}}$. For $\mathrm{n}=6$, we would expect this state to have a mass 128 times that of the proton. Of course that would give the observed $125 \mathrm{GeV}$ resonance. This argument is only a possibility, but is there a theory to prove that $\mathrm{n}=6$ is in fact the size dependence of the gluon field for the $2 p$ state? In the next paragraph, we shall use the $5 \mathrm{D}$ projection model theory $[9,10]$ to show that this is exactly the case, and hence the $125 \mathrm{GeV} 2$ photons emission is totally predictable.

According to the $5 \mathrm{D}$ projection theory, the gluon field is the result from the vector potentials generated by the intermediate quark currents. Thus for the proton, it comes from the product of three vector potentials (see Equationa (4.25) and (4.26) of ref. [9]). Therefore the gluon field $r$ dependence in the proton is $(1 / r)^{3}$. Because the $p-p$ Cooper pair Boson state contains 6 quarks instead of 3 , the gluon field then generated by its intermediate quark state currents must also vary as $(1 / \mathrm{r})^{6}$. Thus if the argument holds that $\mathrm{r}$ is reduced by $1 / 2$ as compared to the proton size, than it is clear that a resonance of the order of $125 \mathrm{GeV}$, that would decay into 2 photons, is expected, since the rest mass of the proton is close to $1 \mathrm{GeV}$. We mentioned earlier that gauge confinement of the quarks suggests that the value of $r$ in the $2 p$ Cooper state can be $1 / 2$ that of the proton. However, gauge transformation is dependent on the imposed vector potential to define the loop integral, yet we have no idea what proper vector potential is involved within these hadrons in question, except we do know that it must be generated by the $u$ and $\mathrm{d}$ quarks motion within these hadrons. Therefore, instead of looking at the charge currents, we look at the angular momenta from the bare masses of the $u$ and d quark constituents. As was shown in [9], these angular momenta must also obey Bohr-Sommerfeld quantization. Hence, by simply comparing the ground state, the fact that the $2 p$ state contains quarks that is twice as massive as those in the $p$ state, it is clear that $r$ of the $2 p$ state is $1 / 2$ of that of the proton. Of course other modifications also play a minor corrective role. For example, the velocity of the quarks within these two hadronic states might be slightly different, etc. None-the-less, it is clear that the 5D projection theory would have predicted such a $125 \mathrm{GeV} 2$ photon emission, hence its interpretation as an indication of the existence of a Higgs field is suggested to be premature.

\section{Conclusions}

Although we have only presented the basic points through the projection operations on the 5D homogeneous spacetime manifold we have shown that we not only can get the standard model, where quarks have mass, but the explicit formulation of the gluon fields and through them the actual values for the meson and baryon masses.

In fact, the gluon fields together with quantum gauge constraint are responsible for the major portion of the hadron mass as discussed previously. It was found that the SU(3) representations for the mesons and baryons together with the mass levels within each representations are generated by the gluon fields strength factors which form their respective Lorentz jet sum rules. Furthermore, we deduce from the meson jet sum rule the remaining mesons, the J/ $\Psi$ particle with the exact mass of 3096 $\mathrm{MeV}$, and the $\mathrm{Y}$ particles with mass $9460 \mathrm{MeV}$ and 4140 $\mathrm{MeV}$. For the baryons, there might be the not yet found octet with mass levels in the 5 to $8 \mathrm{GeV}$ energy region, with mass levels may be also in the $\mathrm{GeV}$ range, far higher than those in the known octet and decuplet.

In conclusion, it was found that the simultaneous gauge and Bohr-Sommerfeld quantization on the quark constituents in hadrons is shown explicitly affecting the gluon potential induced mass levels in the SU(3) representations, as well as the fine mass structure splittings within each mass level, based on the 5D homogeneous space-time projection theory [9]. It was also shown that violating this constraint could lead to inconsistencies with the actual masses of the hadrons. Furthermore, the analysis of the hadron masses leads to the suggestion that the unit quark mass " $m$ " from the 5D homogeneous space-time projection theory is a universal constant, like $e$ and $h$.

We like to emphasize that not only the representations and individual masses of the known hadrons can be numerically deduced from the 5D homogeneous space-time projection model for the establishment of the standard model, but it actually predicts the oscillation of the neutrino, and the finding of a $125 \mathrm{GeV} 2$ photon emission 
state created by the $\mathrm{p}-\mathrm{p}$ collision CERN experiment, without any necessity of introducing a condensed Higgs Boson vacuum state for the universe. In fact for the 5D theory, matter will never totally revert to pure mass-less energy fields, as there is no vacuum phase transition ever possible.

\section{REFERENCES}

[1] A. Cho, "Higgs Boson Makes Its Debut after DecadesLong Search," Science, Vol. 337, No. 6091, 2012, pp. 141-143. doi:10.1126/science.337.6091.141

[2] A. Witze, "Story One: Higgs Boson Fills Last Gap in List of Basic Particles: Little Doubt Left That Physics' Standard Model Is Complete," Science News, Vol. 182, No. 2, 2012, pp. 5-6. doi:10.1002/scin.5591820803

[3] A. Cho, "Last Hurra: Final Tevatron Data Show Hints of Higgs Boson," Science, Vol. 335, No. 6073, 2012, p. 1159. doi:10.1126/science.335.6073.1159

[4] P. W. Higgs, "Broken Symmetries and the Masses of
Gauge Bosons," Physical Review Letters, Vol. 13, No. 16, 1964, pp. 508-509. doi:10.1103/PhysRevLett.13.508

[5] M. Gell-Mann, "Non-Leptonic Weak Decays and the Eightfold Way," Physical Review Letters, Vol. 12, No. 6, 1964, pp. 155-156. doi:10.1103/PhysRevLett.12.155

[6] "Daya Bay Reactor Neutrino Experiment," Wikipedia, 2012.

[7] http://neutrino.physics.berkeley.edu/news/News.html

[8] Anonymous, "Success for China's Neutrino Experiment," Nature, Vol. 483, 2012, p. 250.

[9] K.-W. Wong, G. A. M. Dreschhoff and H. J. N. Jungner, "The Homogeneous 5D Projection and Realization of Quark and Hadron Masses," arXiv: 1202.5761v1, 2012.

[10] K.-W. Wong, G. A. M. Dreschhoff and H. J. N. Jungner, "The J/ $\Psi$ Meson and the Missing Heavy Baryon Octet," arXiv: 1204.0238v1, 2012.

[11] K. Nakamura, et al., "Review of Particle Physics: Quarks," Journal of Physics G: Nuclear and Particle Physics, Vol. 37, 2010, Article ID: 075021.

[12] “Quark,” Wikipedia, 2012. 